Special issue of the 3rd International Conference on Computational and Experimental Science and Engineering (ICCESEN 2016)

\title{
The Metabolic Relationships between Probiotics and Fatty Acids
}

\author{
A. DAYANGaÇ* AND B. ERDEM \\ Ahi Evran University, Art and Science Faculty, Department of Biology, Kirsehir, Turkey
}

\begin{abstract}
Probiotics are alive bacteria that have a healthy effect on the hosts and are found in large quantities in the soil and foods. The most recommended probiotics as commercial are the some species of Lactobacillus sp., Bifidobacterium sp., and Streptococcus sp. The fatty acids are formed by the colonic gut flora from dietary fibres, which manage to escape the host's enzymatic digestive systems in the small intestine. Dietary fibres that manage to reach the large intestine are available for several bacterial fermentative reactions. The fermentation of the different dietary fibres leads to an increase in concentrations of several short-chain fatty acids, especially butyrate, propionate and acetate, in the lumen of the proximal regions of the large intestine. In this review, we acquainted that the relationship between fatty acids and probiotic bacteria affects some various health ailments.
\end{abstract}

DOI: $10.12693 /$ APhysPolA.132.816

PACS/topics: probiotics, fatty acids, microbial ecology

\section{Introduction}

The term probiotic is a relatively new word meaning "for life" and it is currently used to name bacteria associated with beneficial effects for humans and animals. The most recommended probiotics as commercial are the some species of Lactobacillus sp., Bifidobacterium sp. and Streptococcus sp. [1]. The probiotics healthy doses or levels are recommended approximately a minimum of $10^{8}-10^{9} \mathrm{CFU}$ (colony-forming unit) probiotics as daily in the $100 \mathrm{mg}$ or $100 \mathrm{ml}$ nutrients. Lactobacillus and $B i$ fidobacterium are normal inhabitants of the human adult gastrointestinal tract. Selected strains from these genera are found in many dairy products, since these microorganisms are regarded as probiotics due to their capacity to improve some biological functions in the host [2]. During the past two decades probiotic micro-organisms have been increasingly included in various types of food products, especially in fermented milks and yoghurts. Probiotics can metabolize indigestible oligosaccharides and other components (called prebiotics) in the intestine [3].

\subsection{The physiological properties of probiotics}

Probiotic bacteria must have various properties to be accepted as a probiotic bacteria. Firstly, probiotics have $\beta$-D-galactosidase activity and proteolytic activity in order to fermentation [4]. Another functional characteristic of probiotics is to have an acid and bile salt tolerance. The cell membranes of microorganisms consist of lipid and fatty acids and bile salts is important to the viability of microorganisms. Probiotics have a bile salt hydrolysis enzyme and this enzyme can reduce the detergent effect of bile salts [5]. In addition to these features of probiotics, most probiotics have adhesion to the intestinal wall [6]. Probiotic bacteria also create a number of other

*corresponding author; e-mail: alpdayangac@gmail.com acids such as citric acid and hippuric acid. Lactic acid bacteria occur as antimicrobial agents such as hydrogen peroxide $\left(\mathrm{H}_{2} \mathrm{O}_{2}\right)$, diacetyl, and bacteriocins. These substances inhibit some organisms which include to cause food spoilage organisms and pathogens. The substances produced by some bacteria in foods have the opposite effect in terms of the viability of probiotics [7].

\subsection{Antimicrobial properties of probiotics}

Probiotics produces the bacteriocins that have antimicrobial property [8]. Bacteriocins are generally divided into 4 classes according to molecular size, chemical structure, mechanism of action, and heat stability. The first group of bacteriocins include lanthion and the methyllanthion amino acid derivatives. The biochemical structure of this group of bacteriocins have also dehydroalanine and dehydrobutyrine. This group of bacteriocins show antimicrobial activity by inhibiting specific enzymes [9]. The second group of bacteriocins is especially active against listeria [10]. Bacteriocins in the third group have a greater molecular weight and consist of the peptide chain that is sensible to heat. The bacteriocins in fourth group are large and complex molecules, require adequate carbohydrate or lipid component for antimicrobial activities [11]. The mechanisms action of bacteriocins varies according to Gram properties of the bacteria [12].

\section{Fatty acids and probiotics}

The fatty acid is carboxylic acid with a long aliphatic chain, which is either saturated and unsaturated [13]. Modifying ruminal microbial metabolism of fatty acid in rumen through animal diet formulation is an effective way to enhance these functional fatty acids in ruminantderived food products [14].

\subsection{Omega-3 fatty acids and probiotics}

The bioactive food compounds include not only polyunsaturated fatty acids, antioxidants, vitamins, peptides, phytosterols, lutein, lycopene, but also living cells 
such as probiotic bacteria [15]. When probiotic bacteria and omega-3 fatty acids are coencapsulated in a single product, there may be synergistic health benefits. A synergetic effect between omega-3 fatty acids and probiotic bacteria during digestion has been reported, where omega-3 lipids help probiotic bacteria attach to the intestinal wall [16]. Additionally, the embedding of the L. casei in the WPI-GA complex coacervates and the encapsulation of tuna oil in the $L$. casei embedded complex coacervate as capsule shell might have minimised the exposure of $L$. casei 431 cells to the acid stressor and this may have improved the cell viability [17]. Similarly it suggests that probiotics and long chain polyunsaturated fatty acids (LCPUFAs) modulate T-helper 1 and 2 responses, show antibiotic like actions, and alleviate changes related to allergic inflammation [16].

\subsection{The relationship conjugated linoleic acid with probiotics}

Conjugated linoleic acids (CLA) are a family of at least 28 isomers of linoleic acid found mostly in the meat and dairy products derived from ruminants [18]. CLA and the related conjugated linolenic acid (CLNA) isomers are bioconverted from linoleic acid and alpha-linolenic acid, respectively, mainly by Bifidobacterium bacteria strains inhabiting the gastrointestinal tract [19]. Probiotics can produce conjugated linoleic acid (CLA) in ruminants. Lactobacilli, bifidobacteria and propionic bacteria make conjugated linoleic acid from linoleic acids in the gastrointestinal tract [20]. The main source of dietary lipids in animal chow is soy-bean oil, which is composed at $65 \%$ of long-chain polyunsaturated fatty acids. It is well known that Lactobacillus hydrolyzes soy oil to conjugated linoleic acid efficiently which results in a reduction of plasma lipoprotein concentrations and hepatic cholesterol [21].

\subsection{Short-chain fatty and probiotics}

Short-chain fatty acids (SCFAs) are saturated fatty acids featuring an aliphatic chain length of at most eight carbon atoms [22]. The fermentation of the different dietary fibres leads to an increase in concentrations of several short-chain fatty acids, especially butyrate, propionate, and acetate, in the lumen of the proximal regions of the large intestine [23]. The fatty acids are formed by the colonic gut flora from dietary fibres, which manage to escape the host's enzymatic digestive systems in the small intestine. Short-chain fatty acids are mainly produced from poly-, oligo- and fructooligosaccharides, proteins, peptides and glycoprotein precursors by anaerobic micro-organisms [24]. Apart from oral and intravenous administration of short-chain fatty acids or their salt forms, the use of pre- and probiotics may also be considered for attainment of these beneficial effects [25]. In the case of prebiotics, dietary fibres that selectively promote the growth of a particular short-chain fatty acidproducing bacterial specie are administered in order to increase the production of its fermentation products [26].
Especially mammals appear to be able to absorb the synthesised short-chain fatty acids from the colonal lumen and generate energy by oxidation of these substrates. SCFAs seem to be able to stimulate the uptake of water and sodium salts from the lumen of the large intestine, resulting in further reduction of the concentration of osmotically active compounds. Therefore it is very unlikely that SCFAs are able to induce diarrhea [27].

\subsection{The effect of other lipid derivatives of probiotic}

The lipid improvement effect of probiotics has raised interest in recent years. Xiao et al. suggested that a lowfat yoghurt containing Bifidobacterium longum decreased the serum total-cholesterol, LDL-cholesterol and triglycerides (TGs) in 42 subjects after 4 weeks [28]. A previous clinical study reported that a probiotic strain, Enterococcus faecium M74, reduced the serum cholesterol level by $12 \%$ in 43 volunteers after 56 weeks [29]. Ashar et al. reported that the administration of a milk containing Lactobacillus acidophilus was associated with the reduction of total cholesterol [30]. Most clinical trials regarding probiotics have focused on individuals suffering from hypercholesterolemia or hyperlipidemia. There have been few clinical studies on the efficacy of probiotics for TG control. A recent study showed that dual probiotic supplementation (L. curvatus HY7601 and $L$. plantarum KY1032) reduced the average plasma TG levels by $40 \%$ compared with placebo treatment in rats [31]. Ahn et al. indicated that the consumption of two probiotic strains for 12 weeks reduced TGs and increased the apo AV and LDL particle size in hypertriglyceridemic subjects [32].

\section{Conclusion}

Many probiotic studies in the scientific literatures have shown that probiotics in the gastrointestinal tract directly affected the fatty acid levels of the host organism. In addition, probiotics appear to have an effect in the regulation of fatty acids metabolism in the gastrointestinal tract. Besides probiotics occur such as short chain fatty acids and conjugated linoleic fatty acid which are essential fatty acids for health. In coclusion, this review suggest that between probiotics and fatty acid molecules there is an important relationship in terms of both gastrointestinal microbiological importance and host organism health.

\section{References}

[1] R. Fuller, Gut 32, 439 (1991).

[2] E. Solauri, S. Salminen, A.C. Ouwehand, Best. Pract. Res. Clin. Gastroenterol. 18, 299 (2004).

[3] M. Saarela, G. Mogensen, R. Fondén, J. Matto, T. Mattila-Sandholm, J. Biotechnol. 84, 197 (2000).

[4] A. Shihata, N.P. Shah, Int. Dairy J. 10, 401 (2000).

[5] S.E. Gilliand, M. Speck, Appl. Environm. Microbiol. 33, 15 (1977). 
[6] N.P. Shah, Food Technol. 55, 46 (2001).

[7] O. Sağdıç, E. Küçüköner, S. Özcelik, Atatürk Üniv. Ziraat Fak. Derg. 35, 221 (2004), (in Turkish).

[8] I. Helandervon, A. Wright, T. Mattila-Sandholm, Trends Food Sci. Technol. 8, 146 (1997).

[9] F. Atalay, D. Asma, E. Aydogmus, H. Turanci, H. Kaya, Acta Phys. Pol. A 125, 235 (2014).

[10] H. Chen, D.G. Hoover, Comphreh. Rev. Food Sci. Food Safety 2, 82 (2003).

[11] S. Kurt, Ö. Zorba, YŸ̈. Vet. Fak. Derg. 16, 77 (2005), (in Turkish).

[12] B. Erdem, A. Dayangaç, M. Günaydin, Ş. Tulumoğlu, M. Yilmaz, Acta Phys. Pol. A 130, 181 (2016).

[13] C. Beermann, J. Jelinek, T. Reinecker, A. Hauenschild, G. Boehm, H.-U. Klör, Lipids Health Dis. 2, 10 (2003).

[14] M.M. Or-Rashid, T.C. Wright, B.W. McBride, Appl. Microbiol. Biotechnol. 84, 1033 (2009).

[15] P. de Vos, M.M. Faas, M. Spasojevic, J. Sikkema, Int. Dairy J. 20, 292 (2010).

[16] U.N. Das, Nutrition 18, 786 (2002).

[17] S. Lahtinen, A. Ouwehand, S. Salminen, P. Forssell, P. Myllärinen, Lett. Appl. Microbiol. 44, 500 (2007).

[18] S. Banni, Curr. Opin. Lipidol. 13, 261 (2002).

[19] C.P. Van Nieuwenhove, V. Terán, S.N. González, in: Probiotics, Ed. Everlon Cid Rigobelo, 2012, p. 565.

[20] J. Jiang, L. Bjorck, R. Fondén, J. Appl. Microbiol. 85, 95 (1998).
[21] S. Xu, T.D. Boylston, B.A. Glatz, J. Agric. Food Chem. 53, 9064 (2005).

[22] K. Yilancioğlu, H.O. Tekin, S. Cetiner, Acta Phys. Pol. A 130, 428 (2016).

[23] S.E. Pryde, S.H. Duncan, G.L. Hold, C.S. Stewart, H.J. Flint, FEMS Microbiol. Lett. 217, 133 (2002).

[24] L.B. Richards, M. Li, B.C.A.M. van Esch, J. Garssen, G. Folkerts, PharmaNutrition 4, 68 (2016).

[25] M. Roberfroid, J. Nutr. 137, 830 (2007).

[26] J.H. Cummings, G.T. Macfarlane, H.N. Englyst, Am. J. Clin. Nutr. 73, 415 (2001).

[27] A.A. Al-Othman, Int. J. Food Sci. Nutr. 51, 159 (2000).

[28] J.Z. Xiao, S. Kondo, N. Takahashi, K. Miyaji, K. Oshida, A. Hiramatsu, K. Iwatsuki, S. Kokubo, A. Hosono, J. Dairy Sci. 86, 2452 (2003).

[29] P. Hlivak, J. Odraska, M. Ferencik, L. Ebringer, E. Jahnova, Z. Mikes, Bratisl. Lek. Listy 106, 67 (2005).

[30] M.N. Ashar, J.B. Prajapati, Folia. Microbiol. (Praha) 45, 263 (2000).

[31] D.Y. Park, Y.T. Ahn, C.S. Huh, R.A. McGregor, M.S. Choi, World J. Gastroenterol. 19, 274 (2013).

[32] H.Y. Ahn, M. Kim, J.S. Chae, Y.T. Ahn, S. Jae-Hun, C. Il-Dong, L. Sang-Hyun, L. Jong Ho, Atherosclerosis 241, 649 (2015). 